\title{
Abordaje familiar en un caso de intento de suicidio
}

\author{
J. J . J iménez Díaz, A. Villanueva Antón*, X. R. García Soto**, \\ E. J. I beas Cuasante***, L. M. Hernando del Pino**** \\ Médico de Familia C.S. Los Cubł\&S.édico de Familia. Coordinador de la Unidad \\ Docente de Medicina Familiar y Comunitârilzasicólogo. Unidad de Salud Mental \\ Infanto-J uvenit***Médico de Familia. Especialista en Bioética. \\ ****Administrativo Unidad de Salud Mental. Burgos
}

\section{RESUMEN}

SVJ es un joven de 21 años a quien se le realiza abordaje familiar por intento de suicidio. La posi ción del médico de primaria cuando decide inter venir en estos casos es privilegiada pues conoce ya a la familia y puede valorar diferentes aspectos que facilitarán la evaluación y toma ulterior de de cisiones.

En el abordaje del paciente se buscó la partici pación familiar, aprovechando la movilización que la propia crisis produjo, para conseguir un com promiso de colaboración. Tras tres sesiones con to dos los miembros familiares se fijaron objetivos conjuntos: ruptura con el ambiente previo, desblo queo comunicativo, búsqueda de entorno favora ble, etc. La evolución ha sido positiva logrando una mejor situación del paciente y familia para afrontar nuevas crisis.

Llegar a comprender el rol del paciente en esa familia y buscar soluciones no es sólo una técnica sino también un compromiso, una experiencia y una disponibilidad de todos los profesionales im plicados.

Palabras clave: Terapia familiar. Intento de sui cidio. Adolescencia.

\section{Accosted family by a suicide attempt}

\section{ABSTRACT}

SVJ is a 21 years old young man, who is studied by a suicide attempt. The position of the family doctor, when the decision of getting involved is taken, is privileged due to he knows the family and he can appreciate some different aspects that will help in the evaluation and the subsequent decisionmaking.

Family participation was looked for in the pa tient accosting to reach a pledge of collaboration, taking advantage of the reaction caused by the cri sis indeed. After three sessions with all the family members some joint objectives were fixed: to bre ak off with the previous environment, to unfreeze the communication, to look for a favorable enviro ment. The evolution has been positive, obtaining a better situation of the patient and family to face new crisis.

To get to understand the role of the patient in that family and to look for solutions is not only a technique but also a compromise, an experience and a availability to all the professionals involved.

Key words: Family therapy. Suicide attempted. Adolescence.

\section{INTRODUCCIÓN}

La conducta suicida es un serio problema de salud pública en el mundo occidental. La mortalidad anual en España por suicidio se estima en 5,7/100.000 habitantes ${ }^{1}$, siendo entre los jóvenes de
15 a 24 años la segunda causa de muerte. En el estudio de Stenager ${ }^{2}$ el 25,2\% de los intentos de suicidio había consultado al médico de cabecera en el mes previo, siendo el porcentaje de atendidos directamente por psiquiatras el $25,2 \%$. Por patologías, la mayoría de las depresiones (64\%), abuso de 
drogas (57\%) y trastornos de adaptación (77\%) son seguidas por el médico de familia, mientras que las esquizofrenias (78\%) y el abuso de alcohol (63\%) por el psiquiatra. Masson ${ }^{3}$ identifica cuatro contextos de riesgo de suicidio, estrechamente relacionados con el funcionamiento familiar: de abusos sexuales, de carencias afectivas, sociales o educativas, de violencia, y de aislamiento y de relaciones rígidas y disfuncionales. Ante estos datos, parece correcto asumir la postura de Diekstra ${ }^{4}$ que considera fundamental la adquisición por los médicos de familia de técnicas de entrevista, tratamiento y seguimiento de pacientes suicidas, así como de su entorno familiar y social.

En este artículo presentamos un caso de intento autolítico por parte de un joven, encuadrado en un contexto de abuso de drogas y dificultades de comunicación con el entorno familiar y social. El médico de Atención Primaria abordó el tratamiento y seguimiento del paciente suicida con una orientación sistémica, a través de entrevistas familiares, obteniéndose resultados satisfactorios, tanto en términos de evolución personal del paciente, como de refuncionalización de las relaciones familiares.

\section{CASO CLÍNICO}

\section{Motivo principal de consulta}

SVJ es un joven de 21 años, conocido y asistido regularmente por su médico de familia desde 1990. En 1998 requiere ayuda por uso de sustancias tóxicas deseando abandonar el consumo de cocaína inhalada y fumada, cannabis, alcohol y tabaco. Afirma no haber consumido otras sustancias como heroína o drogas de diseño. Inició el consumo en 1996 durante la prestación del servicio militar y desde entonces ha realizado un intento de rehabilitación en Proyecto Hombre, sin resultado positivo por abandono del tratamiento. A principios de 1999 el médico de familia recibe una llamada de la madre del paciente, informando que su hijo y su amiga están ingresados por intento autolítico, por haberse seccionado mutuamente los vasos sanguíneos a nivel de carpos. En ese momento el estado clínico de ambos es estable, encontrándose fuera de peligro.

Ante la llamada de la madre y la situación planteada se aconseja consultar con psiquiatría mientras está ingresado y se decide realizar atención médica del paciente para conocer las posibilidades de intervención en su contexto familiar ${ }^{5}$, citando en consulta también a sus padres y sus tres hermanos. Esta decisión está apoyada por expreso deseo del paciente que manifestó: "con la familia que tengo no hace falta ir al psiquiatra".

\section{Experiencia de la dolencia del paciente y otros miembros de la familia}

Durante la primera entrevista, en la que se completa y actualiza el genograma (Fig. 1), la familia se muestra colaboradora y preocupada, aunque comunicativamente inhibida: "quizás fui duro al decirle lo que pensaba: que despreciaba a la familia...", "las drogas le hacen ver amigos en un lugar donde no los hay y él no se da cuenta", " para nosotros ha sido un golpe fuerte, queremos que cambie", comentan los hermanos; "lo mal que lo hemos pasado... y lo mal que lo estamos pasando", añaden los padres. El médico busca crear un clima de distensión que favorezca la libre expresión colectiva. El paciente índice agradece la presencia y apoyo de sus familiares y reconoce haber llevado una doble vida ante ellos ("el que me echaran de casa por ausentarme todo el fin de semana no me servía, pero el que me hayan recogido tantas veces cuando llegaba hecho un trapo el lunes me ha servido de mucho"), ocultando su problema durante los años anteriores ("tenía miedo de volverles a hablar de las drogas a mis padres y hermanos, no quería angustiarles y tomé la decisión de solucionar yo el problema"). Siente el sufrimiento que les provoca, especialmente a la mayor de sus hermanas, de la que dice con gran carga emocional que ha sufrido repetidas experiencias dolorosas (embarazo prematuro, divorcio y dificultades económicas). Relata dificultades de comunicación con el padre, poco receptivo a sus problemas, reprochándole que cuando buscaba apoyo esquivaba la conversación franca con un simple: "todo te pasa a ti". "Se le ha consentido todo, se ha relacionado con gente mayor y más capeada que él, siempre ha sido muy niño", "la responsabilidad que se le ha exigido ha sido cero, no quería ir con sus amigos de siempre porque eran muy niños", refieren el padre y la hermana.

Repasa sus experiencias en el mundo marginal relacionado con las drogas ilegales, tanto de consumidor como de distribuidor a pequeña escala. En ese entorno inicia una relación sentimental con una chica de su edad, con más experiencia que él en estos ambientes y consumidora habitual de distintas sustancias.

La familia se muestra dispuesta a colaborar en el proceso rehabilitador, sin imponer condiciones al paciente ("queremos que se abra y no se calle, para poder ayudarle", "que nos diga si le quedan deudas pendientes, para saldarlas y que no le estén buscando", "solemos hablar de todo entre los hermanos", "yo le recogí en mi casa cuando le echó mi madre, harta de su desaparición los fines de semana").

\section{CONCRECIÓN DEL CASO}

\section{Enfermedades actuales de los miembros de la familia}

SVJ presenta sutura de los vasos, ligamentos, tendones y músculos seccionados, con inmoviliza- 


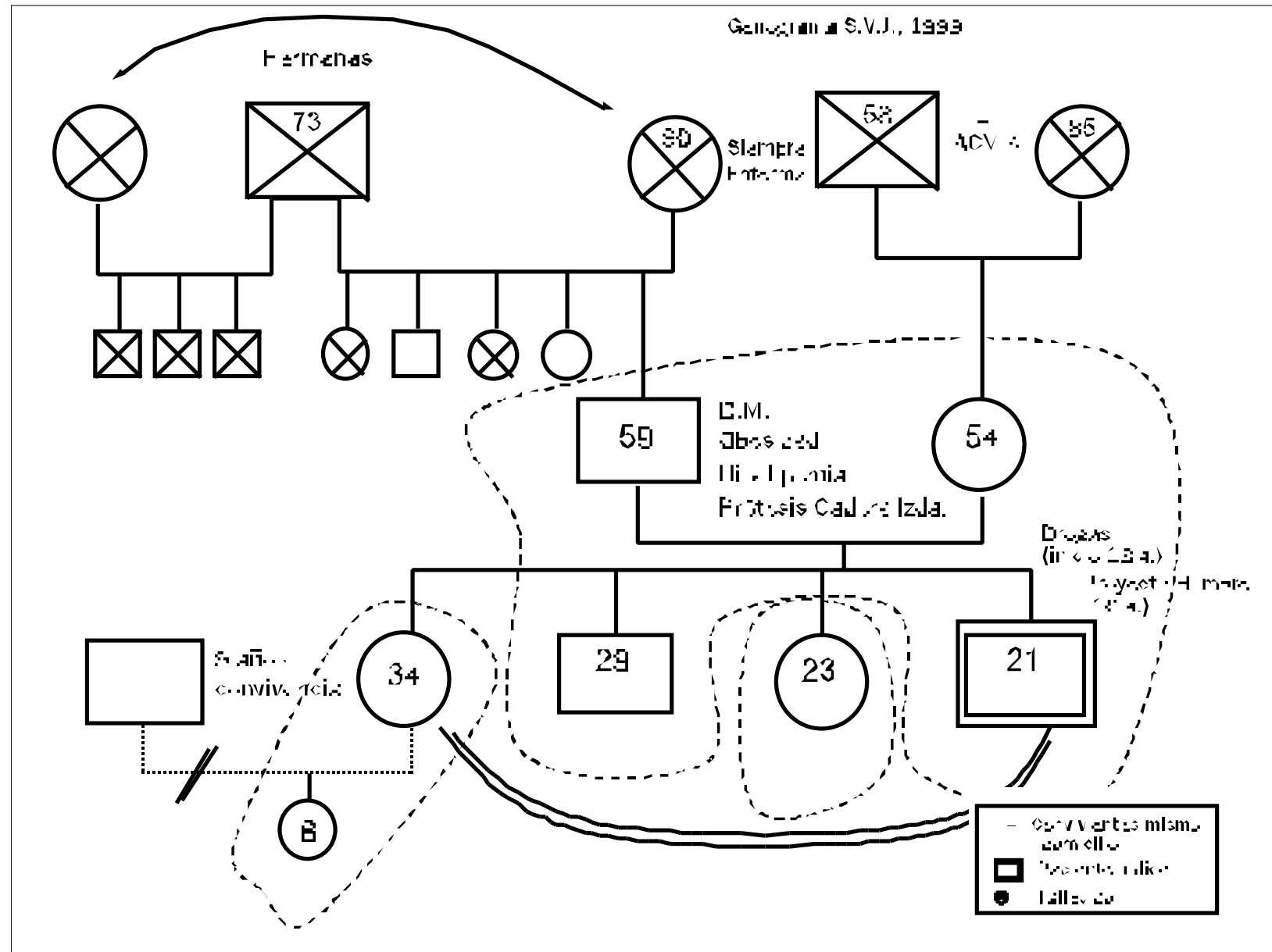

DM : diabetes mellitus

ción por escayola; recibe tratamiento antibiótico. El padre padece diabetes, obesidad e hiperlipemia; portador de prótesis de cadera izquierda, implantada hace ocho años, tras un periodo de incapacidad laboral transitoria de un año, durante el cual fue sometido a control programado de su patología en el centro de salud; tras finalizar el proceso, abandonó todo contacto con el equipo de Atención Primaria; consta consumo excesivo de alcohol, de tipo sociocultural. La madre padece insomnio crónico y cefaleas, consumiendo excesivo café. La menor de las hermanas presenta antecedentes de insomnio, líquen y eczema dishidrótico y psoriasiforme. No constan datos del resto de la familia.

\section{Las personas}

SVJ es el menor de cuatro hermanos. Se muestra extrovertido, inseguro, poco reflexivo y aparentemente irresponsable. Sociable, con tendencia a dejarse arrastrar por el grupo; dependiente de las hermanas y pasivo ante obligaciones. Consta historia de fracaso escolar. En los dos últimos años veía entre 7 y 12 horas diarias de televisión.
La madre manifiesta dificultades para el manejo educativo de los hijos, así como falta de coordinación y acuerdo con su esposo en objetivos educativos; este hecho ya había sido comunicado por el padre en consulta siete años antes. En la entrevista ambos cónyuges siguen de acuerdo con la queja.

El padre aparece con poco papel en la vida afectiva de la familia, explicable en parte por la ausencia del hogar por motivos laborales; no destaca ningún rasgo de personalidad. Despreocupado por su salud. Ante los conflictos familiares tiende a inhibirse o disminuir su importancia.

Se carecen de datos clínicos sobre la hija mayor e hijo segundo.

En la historia clínica de la hija menor constan problemas de sueño y adaptación familiar, así como demanda de atención psicológica, siendo tratada con ansiolíticos y derivada a la unidad de salud mental.

\section{Contexto}

En el hogar conviven los padres y los hijos varones. Las dos hijas abandonaron el hogar familiar a 
los 17 años, manteniendo, no obstante, relaciones fluidas con sus padres. La primogénita mantiene relaciones especialmente estrechas con el paciente. Ambos padres trabajan fuera del hogar. La madre tiene horario de mañana en una empresa de limpieza. El padre es trabajador por cuenta ajena, viaja frecuentemente y está ausente la mayor parte del día.

El paciente cambió de amistades poco antes de abandonar los estudios, dando como motivo que sus amigos de clase eran muy infantiles. Fue entonces cuando se introdujo en el mundo de la droga. A raíz de este cambio, aparecieron frecuentes ausencias del hogar durante los fines de semana. Sin embargo, en todo momento mantuvo buenas relaciones con sus hermanas. Desde los 16 años trabaja de forma discontinua con contratos temporales. En algún caso perdió el trabajo por abandono o como consecuencia de su estilo desordenado de vida. A los 18 años marcha al servicio militar y posteriormente empieza a trabajar de forma estable en una fábrica.

La familia se encuentra en la etapa de ciclo vital correspondiente a la plataforma de despegue o lanzadera $^{6}$, con dos hijas independizadas y dos hijos viviendo en el hogar de los padres, aunque con ingresos propios. Madre menopáusica.

La colaboración con el médico de familia ha sido buena en todo momento.

\section{LISTADO DE PROBLEMAS}

Podemos destacar los siguientes:

1. Consumo de sustancias ilegales.

2. Ambiente marginal con el que se relaciona el hijo; desde el abandono de los estudios ha ido integrándose en entornos de escasa socialización, en los que son habituales patrones de conducta disocial o claramente delictiva.

3. Sentimientos familiares negativos desencadenados por el intento autolítico del hijo menor.

4. Elementos del desarrollo familiar pendientes de resolución: la familia ha ido enfrentándose a una sucesión de estresores importantes a lo largo de la última década con procedimientos no totalmente eficaces; ello produce un sentimiento colectivo de frustración.

Coinciden en este caso algunos de los problemas descritos en la literatura? ${ }^{7}$.

\section{DISCUSIÓN DEL CASO}

\section{Diagnóstico sistémico}

Dentro del sistema familiar se observan debilidades comunicativas en el subsistema conyugal, con relaciones pobres, discrepancias o falta de unidad de criterio educativo hacia los hijos. Las relaciones dentro del subsistema paterno-filial parecen poco definidas, con límites difusos, normas escasamente explicitadas o inexistentes y sin criterios claros de cumplimiento. Cabe señalar que los padres mantienen una actitud abierta a las innovaciones introducidas por el estilo de vida de los hijos.

El subsistema fraternal se caracteriza por relaciones fluidas con respeto a la autonomía personal. El paciente índice se halla estrechamente vinculado a sus hermanas, siendo la menor la más crítica con su comportamiento: "me mintió, me engañó...yo le decía: tú no hables mucho de los drogadictos, porque no sé ni con quién vas".

Las crisis anteriores de la familia no se resolvieron adecuadamente. El abandono del hogar de la primera hija supuso un enfrentamiento conyugal que se resolvió con el tiempo sin ninguna intervención. Cada miembro termina resignándose a aceptar su papel: la madre asumiendo la responsabilidad del seguimiento, la progresiva inhibición del padre y los hermanos definiendo sus papeles sin un modelo de referencia claro y de apoyo adecuado. Probablemente la falta de actuaciones claras en crisis previas sin una jerarquía establecida servía de freno al sistema evitando nuevos desequilibrios. La marcha de la segunda hija fue mejor aceptada, ésta, más conciliadora y dialogante, estableció un plan de vida que discutió con la familia, siendo aceptado.

\section{Diagnóstico estructural}

No parece haber una jerarquía clara en el sistema familiar, favoreciéndolo las ausencias periódicas del padre por motivos laborales y posiblemente también sus rasgos de personalidad. Hay recursos que se movilizan ante el conflicto o amenaza ( $p$. ej., SVJ es acogido en el bar de la hermana mayor para realizar algunas tareas, con el correspondiente control de comportamiento; estancias de fin de semana en el pueblo de origen de los padres).

Retrospectivamente podemos observar la progresiva desestructuración del sistema. A lo largo de los últimos diez años se ha centrado la atención y los recursos familiares sucesivamente en la hija mayor, el padre y la hija tercera, con la consiguiente falta de supervisión del rol, comportamiento y evolución personal del hijo menor.

\section{Proceso familiar}

Estudiando la sucesión de hechos vitales acaecidos a la familia, vemos que la hija mayor abandona el hogar a los 17 años, estableciendo una relación sentimental no formalizada, fruto de la cual nació 
en 1991 una hija; la relación se rompió dos años después.

Entre 1993 y 1994 la hija menor abandonó el hogar sin haber alcanzado la mayoría de edad y el padre pasó un periodo de inactividad laboral, en lista de espera para intervención quirúrgica; durante este tiempo, su papel tradicional en la familia se vio transformado por la permanencia continua en el hogar y la disminución de ingresos. Dados los desacuerdos conyugales antes citados, posiblemente haya resultado estresante para el sistema en su conjunto; es llamativo que sólo durante este periodo haya acudido regularmente al centro de salud, no sólo a recoger los partes de confirmación de baja, sino con buen cumplimiento de todas las citas acordadas en los programas de salud; durante ese periodo comentó con el médico de familia diversos problemas de entendimiento familiar. Tras el alta abandonó totalmente el contacto con el médico.

En 1996 el paciente índice realizó el servicio militar, iniciándose en el consumo de sustancias ilegales.

\section{Estrategia de intervención}

El médico reunió a la familia al alta hospitalaria en tres ocasiones, marzo, abril y junio de 1999. En la primera reunión se intentó conseguir un clima expresivo adecuado y el compromiso y acuerdo de cambio o afrontamiento de los problemas con los siguientes objetivos:

1. Reencauzar a SVJ a un entorno sociocultural saludable, con su aceptación previa:

- Romper con el ambiente marginal y con la chica con la que compartió el intento autolítico, con la que estaba unido por una relación breve.

- Control de llamadas telefónicas e intentos de contacto de los anteriores compañeros.

2. Mejorar la comunicación familiar:

- Mantener al paciente ayudando en el bar de la hermana.

- Verbalización de los conflictos.

3. Estimular el desarrollo personal y familiar de SVJ:

— Se acordó que el paciente índice grabara en cinta sus compromisos (no podía escribir por las lesiones) y lo llevara a la siguiente reunión. Esta cinta fue entregada al médico pocos días después.

4. Cambiar el sentimiento de frustración y dotar a la familia de recursos para la resolución de futuros conflictos:

- Colaboración del médico en el desbloqueo de la comunicación grupal a fin de conseguir que la información se presentara de modo ordenado y comunicativamente aceptable.
- Afianzar la confianza de los familiares en la calidad del apoyo emocional que estaban ofreciendo.

En la siguiente sesión se analizaron los progresos alcanzados: ruptura con su ambiente anterior (incluso el cese de la relación sentimental anterior se produjo antes de lo esperado), buena respuesta laboral del paciente índice, mejoría comunicativa global, aumento de la confianza en sí mismos e iniciativas de tiempo libre conjunto.

Se citó nuevamente al paciente un mes y medio más tarde, acudiendo toda la familia. Se pudo observar entonces una clara mejoría y satisfacción de todos los miembros. Ante la situación no pareció necesario mantener la dinámica de grupo, y las siguientes entrevistas se realizaron a demanda de la familia por problemas sanitarios de otra índole.

\section{EVOLUCIÓN Y SITUACIÓN ACTUAL}

De la información obtenida en posteriores contactos con los miembros de la familia, hasta mayo de 2001 puede deducirse que la evolución de conjunto es satisfactoria.

\section{El paciente}

En sucesivas revisiones se ha constatado un claro progreso de SVJ. Ha ido recuperando las amistades anteriores al periodo problemático. Actualmente está trabajando, tiene hábitos de ocio saludables y proyectos de futuro. Es de destacar su iniciativa social en la formación de un grupo deportivo.

\section{La familia}

Según informa la madre en consulta reciente, la familia funciona de modo cohesionado y ha aumentado sus visitas conjuntas al pueblo de origen. El padre ha acudido a control de diabetes, rompiendo con ello la situación de abandono personal y falta de autocuidado en que se encontraba previamente a la intervención. En cuanto a los hermanos, han aumentado su implicación en el proceso familiar.

CORRESPONDENCIA:

Juan José Jiménez Díaz

C.S. Los Cubos

C/ Lavadores s/n

09003 Burgos

Telf.: 947-270301 


\section{Bibliografía}

1. Martínez de Aragón MV, Llácer A. Mortalidad en España en 1993. Boletín epidemiológico y microbiológico 1997; 4 (22): 177-84.

2. Stenager EN, Jensen K. Attempted suicide and contact with the primary health authorities. Acta-Psychiatric-Scand 1994, 90: 109-13.

3. Masson O. Les suicides des enfants et des adolescents. Schweizerisches Nationalkomitee für Gutstige Gesundheit Arbeitsgruppe für Kriminologie. Verlag Rüeger 1986; 36-75.
4. Diekstra RFW, Van Egmond M. Suicide and attempted suicide en general practitioner: 1979-86. Acta- Psychiatric- Scand 1989; 79: 268-75.

5. McDaniel S, Campbell TL, Seaburn DB. Orientación familiar en Atención Primaria. Barcelona: Springer 1998.

6. Medina E. La familia lanzadera. En: De la Revilla L. Manual de Atención Familiar II. Bases para la práctica familiar en la consulta. Granada: Adhara, SL 1999; 695-705.

7. Suárez Rodriguez T. Intentos de suicidio en la adolescencia. Medifam 1995; 5 (5): 272-7. 